\title{
Knowledge, awareness and perception regarding physiotherapy services among clinical students in Rajarata University
}

\author{
Senarath DMKN ${ }^{1 *}$, Sandamali AAK ${ }^{2}$, Wijekoon $\mathrm{WMMU}^{2}$, Ilangarathne IPSD ${ }^{1}$, \\ Ranaweera SMKA ${ }^{1}$, Koralegedara KIS ${ }^{1}$, Wickramage $\mathrm{SP}^{2}$, Paththinige $\mathrm{CS}^{1}$ \\ ${ }^{\prime}$ Department of Anatomy, Faculty of Medicine and Allied Sciences, Rajarata University. \\ ${ }^{2}$ Department of Physiology, Faculty of Medicine and Allied Sciences, Rajarata \\ University. \\ *kumudunisansalas@gmailcom
}

Patients rely on doctors for referral to allied healthcare services including physiotherapy. Doctors need to be wellinformed about physiotherapy to provide effective patient care in many situations. This study assessed the knowledge, awareness and perceptions regarding physiotherapy among the clinical students in Faculty of Medicine and Allied Sciences, Rajarata University of Sri Lanka (FMAS, RUSL). A descriptive cross sectional study was conducted among clinical students of FMAS, RUSL. Data was collected using a pre-tested, structured, self-administrated questionnaire and analyzed using SPSS-20. Among 84 respondents, all were aware of the presence of physiotherapy profession. Most commonly known areas of physiotherapy specializations were orthopedic (39; 46.4\%), neurology (30; $35.7 \%)$ and pediatric $(23 ; 27.3 \%)$. Majority (92.9\%) were familiar with the use of exercises in physiotherapy, however knowledge regarding other physiotherapy modalities were low.
Commonly known physiotherapy techniques were joint mobilization (94\%), stretching $(91.7 \%)$, soft tissue mobilization (66.6\%) and movement rehabilitation (60.7\%). Most of the students knew the application of physiotherapy in spinal cord injury $(75 \%)$, cerebral palsy $(70.2 \%)$ and stroke $(67.8 \%)$ but it was poor in conditions like torticollis (5.9\%), scoliosis (27.4\%) and burns (26.2\%), where physiotherapy is more applicable. Majority of the students agreed that physiotherapy play an important role in hospitalized patients $(94 \%)$ and community-based rehabilitation (79.8\%). Most of the students perceived inadequacy of knowledge regarding physiotherapy $(72.6 \%)$. The study revealed that the medical students' knowledge and awareness regarding physiotherapy services needs improvement and more effective means of informing medical students about physiotherapy are required. 